\title{
Pharmacological management of panic disorder
}

\author{
Carlo Marchesi \\ Psychiatric Section, Department of \\ Neuroscience, University of Parma, \\ Parma, Italy
}

\begin{abstract}
Panic disorder (PD) is a disabling condition which appears in late adolescence or early adulthood and affects more frequently women than men. PD is frequently characterized by recurrences and sometimes by a chronic course and, therefore, most patients require longterm treatments to achieve remission, to prevent relapse and to reduce the risks associated with comorbidity. Pharmacotherapy is one of the most effective treatments of PD. In this paper, the pharmacological management of PD is reviewed. Many questions about this effective treatment need to be answered by the clinician and discussed with the patients to improve her/his collaboration to the treatment plan: which is the drug of choice; when does the drug become active; which is the effective dose; how to manage the side effects; how to manage nonresponse; and how long does the treatment last. Moreover, the clinical use of medication in women during pregnancy and breastfeeding or in children and adolescents was reviewed and its risk-benefit balance discussed.
\end{abstract}

Keywords: panic disorder, pharmacological treatment, treatment guidelines

\section{Introduction}

Panic disorder (PD) (Table 1) is a disabling condition which exerts a negative impact on social, family and working lives of patients. PD is common in the general population, showing a one year prevalence of $2.7 \%$ and a life-time prevalence of $4.7 \%$ (Kessler, Berglund et al 2005; Kessler, Chiu et al 2005).

PD is consistently found to occur more frequently in females (twice more often in women than in men) (Eaton et al 1998; Kessler et al 1998) and to appear in late adolescence or early adulthood (Weissman et al 1997; Eaton et al 1998; Kessler et al 1998). However, nearly half of adult patients report significant difficulties with anxiety during childhood (Pollack 2005) and PD has frequently been diagnosed in clinicallyreferred children and adolescents (10\%) (Masi et al 2000).

PD is frequently associated with other mental disorders during life-time: mood disorders, in particular major depression (Kessler et al 1998; Simon and Fischmann 2005), anxiety disorders, in particular agoraphobia (Goisman et al 1994), and alcohol abuse (Zimmermann et al 2003) are observed in many PD patients. Comorbid disorders may contribute to increase the severity of the illness, to be associated with suicide attempts, to reduce the response to treatment and to induce relapse (Lecrubier 1998; Slaap and den Boer 2001; Vickers and McNally 2004; Sareen et al 2005; Goodwin and Roy-Byrne 2006; Marchesi et al 2006a).

Also personality disorders, specifically those of the anxious cluster, are commonly observed in PD patients (Massion et al 2002; Grant et al 2005; Marchesi et al 2005). Personality disorders are supposed to increase the severity of symptoms (Ozkan et al 2005), to predict the pattern of comorbidity, particularly the co-occurrence of major depression (Ampollini et al 1999; Ongur et al 2005; Ozkan et al 2005) and to have a negative influence on the response to treatment (Dreessen and Arntz 1998; Slaap and den Boer 2001; Marchesi, De Panfilis et al 2006). 
Table I Diagnostic criteria for panic disorder, according to DSM-IV TR (APA 2000)

I. Recurrent unexpected panic attacks*.

2. At least one of the attacks has been followed by I month (or more) of one ( or more) of the following:

- persistent concern about having additional attacks;

- worry about the implication of the attack or its consequences;

- a significant change in behaviour related to the attacks.

3. The panic attacks are not due to the direct physiological effects of a substance or a general medical condition.

4. The panic attacks are not better accounted for by another mental disorder, such as social phobia, obsessive-compulsive disorder, posttraumatic stress disorder, or separation anxiety disorder.

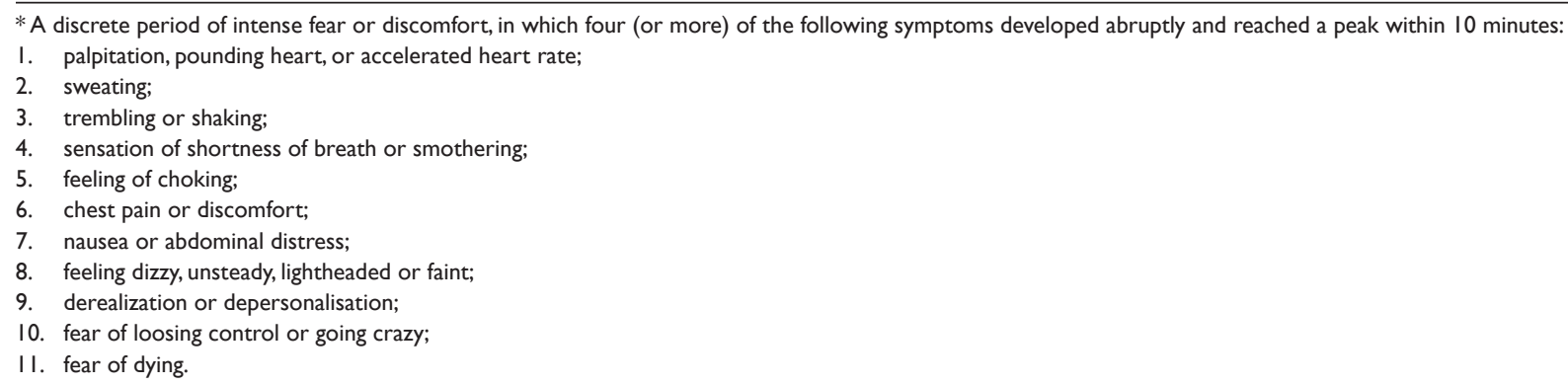

The course of PD is variable: approximately one third of patients achieve remission, whereas one out of five follows an unremitting and chronic course (Rosenbaum et al 1996; Katschnig and Amering 1998). Therefore, most of PD patients require long-term treatments (Davidson 1998; Doyle and Pollack 2004) and continuous evaluations to achieve remission, to prevent relapse and to reduce the risks associated with comorbidity.

Since PD mimics many life-threatening conditions (ie, cardiac or respiratory diseases) or is often associated with a catastrophic misinterpretation of physical sensations, PD induces a high use of medical services, particularly emergency departments (Katerndahl and Realini 1995; Ballenger 1997; Marchesi et al 2001; Marchesi et al 2004). Although, PD is frequently the reason for seeking treatment for medically unexplained symptoms (Kroenke 2003), it also increases the use of medical care when associated with medical illness and the risk of death in patients affected by coronary heart disease (Simon and Fischmann 2005).

Therefore, the identification of this disorder and the appropriate treatment is essential to improve the quality of assistance and to reduce the waste of health care resources through unnecessary medical care. Educational efforts will ensure that effective treatments will be available for a greater number of patients.

The aim of this review is to provide clinicians with the useful information concerning the pharmacological treatment of PD, particularly the choice of medication, the management of non-responders, to decide when and how to discontinue treatment and to balance the risks and benefits in treating PD in women during pregnancy and breastfeeding.

\section{Treatment}

\section{General considerations}

Excluding medical disease

A careful history and physical examination are necessary for all patients to rule out medical causes of symptoms. Hyperthyroidism and hypothyroidism, pheochromocytoma, temporal-lobe epilepsy, asthma, cardiac arrhythmias, excessive intake of caffeine or other stimulants, withdrawal from alcohol, and treatment with high doses of corticosteroids can mimic or cause panic attacks.

\section{Assessment of comorbidity}

Particular attention should be paid in evaluating the presence of depressive symptoms in properly treated patients and to prevent the suicide risk, keeping in mind that depressed PD patients are less likely responders to treatment than non depressed subjects (Slaap and den Boer 2001; Marchesi et al 2006a).

Moreover, the use of alcohol as self-medication must be screened, the abuse should be prevented and the withdrawal symptoms should be properly treated. Finally, the evaluation of personality disorders is clinically useful, because these disorders may be associated with poor compliance and poor response to treatment (Slaap and den Boer 2001; Marchesi, De Panfilis et al 2006).

\section{Education of patients and their families}

Clinical experience suggests that patients and their families benefit from education about PD as the cause of symptoms and about the mechanisms by which this disorder may provoke physical symptoms. In patients, this information 
may alleviate fears of having a serious condition, worries about the physical consequences of the attacks, and may convince families that the patient is affected by a disorder, thus avoiding the behavioral anger or rejection.

\section{The outcome of treatment: response and remission}

Panic attacks are the core feature of PD, yet other symptoms and functional impairment in daily life must be taken into account in evaluating the outcome of treatment. Therefore, the assessment of limited symptom panic attacks, anticipatory anxiety, phobic avoidance and depression is fundamental during treatment and the clinician must carefully monitor treatment to ensure that an optimal response is achieved.

However, no consensus exists regarding the standard criteria of response and remission, because there is no agreement about symptom level resolution and the time frame to ensure stability. According to DSM-IV criteria, patients have been defined as "panic free" if they do not have a sufficient number of panic symptoms to meet the diagnostic criteria of PD. However, the "panic free" definition does not necessarily mean being free of all panic symptoms. In order to provide a frame of reference to clinicians, the International Consensus Group on Depression and Anxiety (ICGDA) (Ballenger et al 1998) suggests that the outcome of treatment should be evaluated in five domains: panic attacks (including limited-symptom attacks), anticipatory anxiety, panic-related phobias, overall illness severity and disabilities. The ICGDA defines: (1) "response" as a stable clinically significant improvement of the full range of symptoms, but more than minimal symptoms continue to be present; and (2) "full remission" as the resolution of all five domains of symptoms, maintained for a period of three months.

Even though the ICGDA recommends the careful evaluation and treatment of depression, it was not included in the criteria to define response or remission. However, a few years later, the definition of $\mathrm{PD}$ remission also included the resolution of depressive symptoms (Ballenger 1999, 2001; Doyle and Pollack 2003).

However, in many clinical trials, the decrease of the frequency of panic attacks or global severity of anxiety has been used as primary efficacy endpoints and has been evaluated over a short-term period (6-12 weeks) (den Boer 1998; Mitte 2005).

Therefore, the conclusions about the efficacy of treatment drawn from the data of clinical trials can be extended to patients treated in clinical practice with some limitations, because these two populations show clinical differences.
In fact, a higher rate of comorbidity, a more severe disorder and a lower level of compliance are frequently observed in patients treated in a clinical setting compared to patients enrolled in clinical trials. Moreover, most clinical trials provide a short-term evaluation of treatment, whereas PD patients require long-term treatment to achieve remission. Finally, the criteria of response often used in clinical trials (50\% decrease in the frequency of panic attacks or global anxiety) (Mitte 2005) are associated with persistence of some anxious and depressive symptoms (Marchesi et al 2006a) and with an impairment in social functioning.

Clinicians and patients should be aware that PD requires long-term treatment to achieve remission, to prevent relapse and to reduce the risks associated with comorbidity.

\section{Effective treatments}

Currently, available clinical practice guidelines for the treatment of $\mathrm{PD}$ recommend pharmacotherapy or cognitive behavioral treatment (CBT), as effective treatments.

These recommendations have recently been confirmed by a meta-analysis, comparing the efficacy of CBT, pharmacotherapy and placebo in PD (Mitte 2005). The metaanalysis evaluated 53 studies of drug treatment (including a total of 7725 patients) and 43 studies of CBT (including 1811 patients). Pharmacotherapy and CBT was superior to placebo, and showed a similar effect in alleviating anxiety, agoraphobia, depression and poor quality of life.

However, some differences exist among available guidelines regarding the recommended first choice treatment:

1. both the American Psychiatric Association (APA 1998) and the British Association for Psychopharmacology (BAP) (Baldwin et al 2005) recommend either pharmacotherapy or CBT because "there is no convincing evidence that one modality is superior for all patients or for a particular subpopulation of patients" (APA 1998, p 2), and "drug and psychological treatments, delivered singly, have broadly similar efficacy in acute treatment" (BAP 2005, p 580);

2. the National Institute of Clinical Excellence (NICE 2004) recommends to consider that $\mathrm{CBT}$ has an effect that lasts longer than that of drugs;

3. the Royal Australian and New Zealand College of Psychiatry (RANZCP 2003) recommends CBT as "the most consistently efficacious treatment" (p 645). This conclusion has been also supported by the observation that CBT can "reduce the probability of relapse" (the same effect has not been demonstrated for pharmacotherapy) (p 644). 
Regardless of the differences among guidelines, patients and their families must be advised about the advantages and the disadvantages of these two effective treatments (Table 2), to provide them with the correct information necessary to participate in the decision regarding treatment.

\section{Pharmacological treatment Neurobiological bases of the pharmacological effect}

Consistent with the hypothesis that a dysfunction of brain pathways (Ninan and Dunlop 2005), using $\gamma$-aminobutyric acid (GABA (Zwanzger and Rupprecht 2005), serotonin (Maron and Shlik 2006) and noradrenaline (Neumeister et al 2005) as neurotrasmitters, is involved in the pathogenesis of $\mathrm{PD}$, the efficacy of antipanic drugs is probably mediated by their effects on these neurochemical systems, even though their exact mechanism remains unclear.

\section{The clinical strategies in pharmacotherapy}

Once pharmacotherapy has been chosen, many questions about this effective treatment need to be answered by the clinician and discussed with the patients to improve his or her adherence to the plan of treatment; it is important to determine the drug of choice, to establish when the drug becomes active, what is the effective dose, how to manage side effects and non response, and the duration of treatment.

\section{The choice of the drug Efficacy \\ Placebo effect}

The placebo effect is a well-known phenomenon in the treatment of PD. The percentage of patients who achieved a free from panic attacks status after placebo treatment ranged from $14 \%$ to $59 \%$ in controlled clinical trials using SSRI, TCA or BDZ as active treatment (den Boer 1998). In a recent study (Khan et al 2005), the mean percentage of symptom improvement was $50 \%$ in 1157 PD patients treated with placebo. Consequently, the aforementioned data suggest that a placebo plays an important role effect in short-term treatment of PD patients. However, the placebo effect disappears with time, and thus the available guidelines recommend the administration of medication that is more effective than placebo.

\section{Effective medications}

Four classes of drugs are more effective than placebo and showed similar efficacy in treating PD: tricyclic
Table 2 Advantages and disadvantages of pharmacotherapy and cognitive-behavior therapy (CBT) in the treatment of panic disorder

\begin{tabular}{lll}
\hline & Pharmacotherapy & CBT \\
\hline Side-effects & ++ & 0 \\
Rapid onset of action & $++^{*}$ & + \\
Efficacy on more severe panic & & + \\
$\quad$ attacks and anticipatory anxiety & ++ & ++ \\
Efficacy on phobic avoidance & + & + \\
Efficacy on severe depression & $++^{\S}$ & \\
$\begin{array}{l}\text { Persistence of effect after } \\
\text { stopping treatment }\end{array}$ & 0 & + \\
Use in primary care setting & ++ & 0 \\
$\begin{array}{l}\text { Used by experienced therapist } \\
\text { Need to perform "homework" }\end{array} \quad+$ & ++ \\
or to confront feared situations & 0 & ++ \\
\hline
\end{tabular}

Note:*Particularly benzodiazepines or $\S_{\text {antidepressants. }}$

antidepressants (TCA), selective serotonin reuptake inhibitors (SSRI), monoamine oxidase inhibitors (MAOI) and high-potency benzodiazepines (BDZ).

In a recent meta-analysis (Mitte 2005) the efficacy of TCA, SSRI and BDZ was compared: 53 studies were analyzed for a total of 7725 patients. TCA, SSRI and BDZ showed a similar effect in improving anxiety (symptoms and frequency of panic attacks) and agoraphobia, whereas SSRI and TCA were superior to BDZ in alleviating depression.

Since the efficacy of all four classes of medications are roughly comparable, the criteria for choosing medication for PD patients mainly depend on considerations of adverse side effects (Table 3 ). Therefore, all the available guidelines recommend an SSRI compound as a first choice drug for the treatment of PD, given the beneficial side-effect profile of this class of drugs.

\section{Advantages and disadvantages of different classes of drugs SSRI}

Six SSRIs are now available: citalopram. es-citalopram, fluoxetine, fluvoxamine, paroxetine and sertraline. No evidence suggests a differential efficacy within the SSRI class, whereas differences exist in side-effects profiles, drug interaction and half-life (Table 4).

Advantages. The SSRI, compared to TCA compounds, are characterized by:

1. a low affinity for the muscarinic, $\alpha_{1}$-adrenergic and histaminergic receptors and therefore rarely induce blurred vision, dry mouth, increased heart rate, constipation, urinary hesitancy, orthostatic hypotension, sedation and weight gain; 
Table 3 Advantages and disadvantages of the different classes of drugs commonly used in the treatment of panic disorder

\begin{tabular}{llll}
\hline & SSRI & TCA & BDZ \\
\hline Rapidity of effect & + & + & +++ \\
Antidepressant efficacy & +++ & +++ & 0 \\
Efficacy in anxiety comorbidity & +++ & +++ & 0 \\
Sedation & 0 & +++ & ++ \\
Cognitive impairment & 0 & + & ++ \\
Anticholinergic effects & 0 & ++ & 0 \\
Orthostatic hypotension & 0 & ++ & 0 \\
Hyperstimulation & ++ & + & 0 \\
Nausea & ++ & 0 & 0 \\
Weight gain & $0 /+$ & ++ & 0 \\
Sexual dysfunction & ++ & + & 0 \\
Physical dependence & 0 & 0 & ++ \\
Discontinuation symptoms & + & + & +++ \\
Risk of abuse & 0 & 0 & ++ \\
Cardiovascular effects & 0 & ++ & 0 \\
Safety in overdose & 0 & ++ & 0 \\
Drug interaction & $0 /++$ & 0 & 0 \\
\hline
\end{tabular}

2. a safety for serious cardiac effects (Roose and Miyazaki 2005);

3. a low risk after an overdose (Barbey and Roose 1998).

Moreover, SSRIs are not associated with tolerance or physical dependence even though a withdrawal syndrome has been observed when an SSRI is abruptly discontinued (see below).

Finally, SSRIs should be preferred in PD patients with obsessive-compulsive disorder or in patients suffering from other anxiety disorders (Ballanger et al 1998). Given the beneficial side-effect profile, the use of SSRIs has been expected to reduce the attrition rate. A meta-analysis of 43 studies (Bakker et al 2002) showed that the rate of drop-out was lower in patients treated with SSRI $(18 \%$ out of 1059$)$ than in patients receiving TCA ( $31 \%$ out of
1308). The authors concluded that the lower drop-out rate in SSRI studies is most probably due to the fact that there are fewer side effects which induce patients to abandon the treatment (even though other reasons may contribute to the discontinuation of pharmacotherapy). However, a recent meta-analysis (Mitte 2005) found a similar drop-out rate $(23 \%)$ in patients treated with an SSRI or a TCA, with side effects being the major reason (11\%) for abandoning pharmacotherapy. This result confirmed the data of the two previous meta-analyses conducted by Otto et al (2001) and by RANZCP (2003). Therefore, the use of an SSRI does not seem to be associated with a significant reduction of the discontinuation of treatment compared to a TCA, although the SSRIs side-effect profile should increase the tolerability. This finding points out the need to properly manage the side effects of SSRIs.

Disadvantages. The increased serotonergic activity induced by SSRIs is the cause of side effects such as anxiety, agitation, insomnia, tremors, nausea, anorexia, headache and sexual dysfunction. Since side effects occur early in treatment, before the therapeutic effect, and many PD patients are hypervigilant regarding SSRI side effects, all strategies that minimize them, such as a low starting dose and a short-term co-administration of benzodiazepines are warranted (see below).

Some SSRIs (fluoxetine, fluovoxamine and paroxetine) exert an inhibitory effect on the cytochrome P450 enzymatic system (particularly the isoenzymes 1A2, 2C9, 2D6 and 3A4) (Greenblatt et al 1998); therefore these compounds are associated with potential harmful drug interactions (ie, $\beta$-blockers, some BDZ).

Moreover, even though SSRI administration is not associated with physical dependence, an abrupt discontinuation of the drug may induce a withdrawal syndrome (Zajecka et al 1997; Warner et al 2006). This syndrome is more likely to

Table 4 Differences among the SSRI medications

\begin{tabular}{|c|c|c|c|c|c|c|}
\hline & Citalopram & Escitalopram & Fluoxetina & Fluvoxamina & Paroxetina & Sertraline \\
\hline Anticholinergic effects & 0 & 0 & $0 /+$ & 0 & $0 /+$ & 0 \\
\hline Sedation & $0 /+$ & 0 & 0 & $0 /+$ & $0 /+$ & 0 \\
\hline Insomnia & $0 /+$ & + & + & $0 /+$ & + & + \\
\hline Orthostatic hypotension & 0 & 0 & 0 & 0 & 0 & 0 \\
\hline Hyperstimulation & + & + & ++ & + & ++ & + \\
\hline Nausea & + & + & + & + & + & + \\
\hline Weight gain & $0 /+$ & 0 & 0 & 0 & + & $0 /+$ \\
\hline Sexual dysfunction & + & + & + & + & ++ & + \\
\hline Discontinuation symptoms & + & + & $0 /+$ & + & ++ & + \\
\hline Cardiovascular effects & 0 & 0 & 0 & 0 & 0 & 0 \\
\hline Safety in overdose & $0 /+$ & $0 /+$ & $0 /+$ & $0 /+$ & $0 /+$ & $0 /+$ \\
\hline Half-life (longer than 24 h) & + & + & +++ & 0 & 0 & + \\
\hline Inhibition cytochrome P450 & 0 & $0 /+$ & ++ & ++ & + & 0 \\
\hline
\end{tabular}


affect patients treated for several months with a short half-life compound, and after a short period of tapering (a few days to few weeks). The withdrawal syndrome begins a few days after discontinuation and is characterized by dizziness, incoordination, headache, irritability, nausea and insomnia, and tends to resolve spontaneously within 1-2 weeks.

\section{TCA}

Imipramine was the first drug used in the treatment of PD (Klein 1964) and along with clomipramine has been the most studied TCA compound in the pharmacotherapy of PD (Bandelow et al 2002, Pollack, Allgulander et al 2003).

Advantages. TCA do not offer major advantages compared to SSRI compounds, while they possess more disadvantages than the latter class of medications.

Disadvantages. The use of TCA, compared to SSRI, is associated with less tolerability and safety, because TCAs can induce several side effects due to their antagonistic effect on muscarinic, $\alpha_{1}$-adrenergic and histaminergic receptors, their effect on cardiac rhythm, and possible harmful consequences after overdose in suicide attempts (Table 3). Moreover, TCAs can induce, more frequently than SSRIs, sweating, weight gain and cognitive disturbances. Finally, like SSRIs, a withdrawal syndrome can occur after an abrupt discontinuation of TCAs (Warner et al 2006).

\section{MAOI}

Irreversible MAOI (phenelzine or tranylcypromine) are generally reserved for patients who do not respond to other treatments, because of their serious side-effects (particularly hypertensive crisis), the harmful consequences in association with other drugs that increase monoamines (sympathomimetic and serotonergic compounds) and the need for dietary restriction (tyramine rich foods increase the risk of hypertension) (Riederer et al 2004). Therefore, irreversible MAOI are considered second-line compounds, used by experienced psychiatrist (APA 1998; ICGDA 1998, Bandelow et al 2002; CPA 2006).

The data about the efficacy of the reversible MAOI meclobemibe are inconsistent and it should be used as thirdline drug (Bandelow et al 2002; CPA 2006).

\section{Benzodiazepines}

The high-potency BDZ (such as, alprazolam, clonazepam, lorazepam) are an effective medication in the treatment of PD (Susman and Klee 2005).

Advantages. In the past decade, BDZ remained one of the most prescribed medications for the treatment of PD. In fact, BDZ was the only treatment used during a follow-up period of 10 years in more than one third of PD patients (Bruce et al 2003). The rapid onset of action, the high tollerability and the high patient acceptance are probably the reasons why BDZ are so often used.

However, the use of these drugs presents some disadvantages compared to the prescription of other effective medications (Table 3).

Disadvantages. On the one hand, BDZ is less effective than TCA or SSRI in treating depression associated with PD (Mitte 2005), and, on the other hand, side effects, such as sedation, somnolence, drowsiness, cognitive impairment, impairment of motor coordination, and the risk of developing tolerance and dependence are limitations for the use of BDZ (Susman and Klee 2005).

A withdrawal syndrome (Schweizer and Rickels 1998) may appear even after short-term administration of BDZ (less than 4 months) and its severity depends on the dose, half life, potency of the drug used, duration of the treatment and the length of the taper period (2-3 weeks recommended after 2 months of treatment). Moreover, PD patients may have difficulties to withdraw from BDZ, probably due to the reappearance of panic attacks triggered by withdrawal symptoms, particularly during a too short period of tapering.

Finally, abuse of BDZ may be found in patients with a history of alcohol or substance abuse, in patients with personality disorders or in patients who use BDZ and alcohol as self-medication. Therefore, in these patients, the use of BDZ should be evaluated with caution and, if possible, avoided.

For these reasons, the available guidelines do not recommended BDZ as the drug of first choice in the treatment of PD.

When recommending the use of a $\mathrm{BDZ}$. The APA (1998) guidelines recommend the combination of SSRI and BDZ in the first weeks of treatment. This recommendation is justified by two clinical observations:

1. some PD patients are very sensitive to the "activation" side effect of SSRI medications and anxiety, agitation, insomnia may appear or increase after administration of the antidepressant, inducing patients to discontinue treatment because medication worsens his or her symptoms;

2. an SSRI compound takes time (4-6 weeks) to become effective.

Therefore, the co-administration of BDZ in the first weeks of treatment may alleviate the "activation" induced by the SSRI and reduce severity and frequency of panic attacks, anticipatory anxiety and/or insomnia (particularly in patients with nocturnal panic attacks) before the SSRI become effective. The co-administration of BDZ is particularly useful 
in patients for whom a very rapid control of symptoms is critical.

A recent study (Goddard et al 2001) confirms the APA guideline recommendation. This study evaluated whether the improvement of panic symptoms was more promptly observed in patients treated with a combination of clonazepam (0.5 mg three times a day) with sertraline than in patients treated with sertraline and placebo. The response rate (defined as a $50 \%$ reduction of the baseline severity) was higher in patients who received the clonazepam-sertraline association than in those who received the placebo-sertraline co-treatment, both after one and three weeks of therapy ( $41 \%$ vs $4 \%$ and $63 \%$ vs $32 \%$, respectively). Similar results were obtained with a clonazepam-paroxetine combination treatment which induced a more rapid response than with the SSRI alone (Pollack, Simon et al 2003).

Therefore, an initial combined treatment followed by BDZ tapering after a few weeks may provide early benefit while avoiding the potential adverse consequences of longterm BDZ use.

Many patients use BDZ "as needed", prior to the exposure to fear situations. An infrequent as-needed use, such as once a week, is not a matter of concern, whereas a more frequent use is not recommended (Zamorski and Albucher 2002).

\section{Other effective drugs}

In a recent study (Bradwejn et al 2005), the extended-release form of venlafaxine (a Serotonin Noradrenergic Reuptake Inhibitor compound) was found to be superior to placebo in reducing the frequency and severity of panic attacks, anticipatory anxiety and phobic avoidance (even though the panic free status was not increased by the compound).

Medications such as trazodone, nefazodone, mirtazapina, reboxetine are recommended (RANZCP, World Federation of Societies of Biological Psychiatry [WFSBP], APA, BPA and CPA guidelines) as second choice drugs because there are only limited data about their efficacy in the treatment of PD.

\section{Ineffective drugs}

The RANZCP, WFSBP, APA, WCA, BPA and CPA guidelines do not recommend the use of medications such as buspirone, $\beta$-blocker propanolol, clonidine, inositol, antihistamines in the treatment of PD, because they are not more effective than placebo.

\section{How to start medication}

A low dose is recommended at the beginning of treatment (Table 5) to minimize the onset and severity of side-effects
(APA 1998; Ballenger et al 1998; Baldwin et al 2005). The dose should then be slowly titrated upward to reach the recommended effective dose (Table 5).

In the first weeks (4-8) of treatment with a SRRI or TCA compound, a concomitant administration of BDZ may be useful to improve clinical condition or to reduce the "activation" side-effects of SRRIs and TCA.

\section{How to manage side-effects}

Patients should be informed about the frequency and the time of onset of side-effects, reassured about the safety profile of the medications (side-effects are troublesome but rarely have harmful physical consequences), and about the decrease of their severity as treatment is continued (the side-effects are usually short-lived).

A common mistake is to begin with a dose that is too high. This increases the risk of onset of side-effects. Therefore, the treatment strategy "start low" and "go slow" improves the tolerability of medication and reduces the discontinuation of treatment because of troubling side-effects.

The persistence of side-effects may be attenuated after switching to a compound of a different class. The intra-class switch is usually not beneficial in reducing side-effects characteristic of a particular class. However, switching to a different SSRI drug may at times be a useful treatment strategy in patients who do not tolerate side-effect despite of partial response (ICGDA 1998; Zamorski and Albucher 2002).

Moreover, an adjunctive therapy, instead of changing an effective medication, might be prescribed to counteract intolerable or persistently severe side-effects. That is, BDZ to manage agitation, anxiety and insomnia; a $5 \mathrm{HT}_{3}$ receptor

Table 5 Doses of effective medication in the treatment of panic disorder

\begin{tabular}{llll}
\hline & mg/day & & \\
\cline { 2 - 4 } & $\begin{array}{l}\text { Starting } \\
\text { dose }\end{array}$ & $\begin{array}{l}\text { Therapeutic } \\
\text { dose }\end{array}$ & \\
\hline Citalopram & 10 & $20-60$ & Once a day \\
Escitalopram & 5 & $10-30$ & Once a day \\
Fluoxetine & 10 & $20-60$ & Once a day \\
Fluvoxamine & 25 & $100-300$ & Bis intra day \\
Paroxetine & 10 & $20-60$ & Once a day \\
Sertaline & 25 & $50-200$ & Once a day \\
Clomipramine & 25 & $75-250$ & Once a day \\
Imipramine & 25 & $75-300$ & Once a day \\
Alprazolam & 0.75 & $2-9$ & Three times a day \\
Clonazepam & 0.75 & $1.5-4.5$ & Three times a day \\
Lorazepam & 1 & $2-7.5$ & Three times a day \\
\hline
\end{tabular}


antagonist (low dose of mirtazapine) to reduce nausea, or a $5 \mathrm{HT}_{2}$ receptor antagonist (low dose of mirtazapine or nefazodone) for sexual dysfunction (Mathew et al 2001; Zamorski and Albucher 2002).

\section{How to manage non-response Definition of non-response}

The outcome of successful treatment should be a stable full remission of all PD symptoms, including depression. Despite progress in the treatment of PD, a significant number of patients continue to suffer from debilitating symptoms, such as panic attacks, phobic avoidance or anticipatory anxiety. A large number of patients do not respond to standard first-line treatments, since $45 \%$ of patients treated with a SSRI in controlled trials failed to achieve a panic free status (Otto et al 2001) and about $40 \%$ of patients were resistant to the combination of medication and CBT (Barlow et al 2000). Therefore, the management of the resistance to treatment in $\mathrm{PD}$ is a frequent problem in clinical practice.

However, before defining a patient as treatment-resistant, the adequacy of treatment should be assessed. In fact, the major reasons for treatment failure can be:

1. the use of ineffective treatments: ie, one study observed that only $39 \%$ of PD patients received a treatment with effective medications or CBT (Taylor et al 1989);

2. the use of inadequate treatment in terms of dose and duration: a large proportion $(47 \%-60 \%)$ of $\mathrm{PD}$ patients received such a treatment (Yonkers et al 1996; Cowley et al 1997);

3. medication intolerance which limits the dose of drugs: ie, intolerable side-effects were the reason for discontinuing medication in $27 \%$ of PD patients in the study of Cowley et al (1997).

Aside from the aforementioned reasons for treatment failure, only one fourth of PD patients reported a lack of benefit after medication (Cowley et al 1997). Therefore, "true" resistance to treatment should only involve a minority of PD patients, when an effective medication is used, the initial dose is low and is slowly increased, and the dose and the length of treatment (see below) are adequate. Obviously, the rate of patients showing a non-response to treatment depends on its definition: ie, the rate of response decreases when the outcome of treatment is the achievement of a stable complete remission of panic attacks (including limited-symptom attacks), anticipatory anxiety, panicrelated phobias, disabilities and depression (Ballenger et 1998). The use of the abovementioned criteria is associated with a remission rate of $46 \%$ in patients treated with a recommended medication for one year in clinical practice (Marchesi et al 2006a).

\section{How much time should one wait before changing an ineffective treatment?}

It is generally accepted that a response to medication does not occur for at least 4 weeks, and some patients do not experience a full response for 8-12 weeks. Therefore, an adequate treatment requires 8 to 12 weeks to obtain a clinically significant improvement.

There is no controlled data to indicate when the treatment should be changed in patients who do not show improvement after pharmacotherapy. WFSBP guidelines (Bandelow et al 2002) suggest that medication should be changed in patients who do not respond after four to six weeks of treatment with a first-choice drug at an adequate dose, whereas if a partial response occurs in the same period of time another four to six weeks of therapy may be required to achieve response. Other guidelines (APA 1998; ICGDA 1998; NICE 2004) suggest that eight to twelve weeks should elapse before changing medication, administered at an adequate dose, when a significant improvement does not occur.

Nevertheless, clinicians are urged by the patients and their families to change ineffective treatment after the first few weeks of therapy without further delay. In a survey of psychiatrists (Scott et al 1999), 78\% of the participants claimed that they would intervene on the treatment regimen when symptom improvement was not observed after 6 weeks of administration of a SSRI compound.

\section{Predictors of nonresponse}

After ruling out that the PD diagnosis is incorrect or due to a general medical condition, the patient is not compliant and the concomitant medications do not have a negative effect on antipanic drugs (ie, pharmacokinetic interaction) (Mathew et al 2001; Bandelow et al 2002), the clinician should evaluate the presence of all the factors known to negatively affect response to pharmacological treatment. In fact, predictors of non-response to medications in PD patients are consistently identified as: younger age at onset, longer duration of illness, higher pre-treatment severity, particularly of phobic avoidance, comorbid major depression, particularly the recurrent form, comorbid anxiety disorders and comorbid personality disorders (Slaap and den Boer 2001, Marchesi et al 2006a, 2006b; Marchesi, De Panfilis et al 2006; Marchesi et al 2007). In $\mathrm{PD}$ patients presenting such conditions, the treatment should be continuously monitored and a long-term duration should be planned. 


\section{Strategies to manage non-response}

The management of patients refractory to medication is not an easy problem to solve in clinical practice because there are no proven effective strategies due to the a paucity of studies that have investigated this topic. This is the reason why some guidelines (RANZCP, APA, WCA) do not include any specific section referring to the management of treatment resistance.

Nevertheless, clinical experience and preliminary reports suggest some strategies to manage resistance to drug.

\section{Pharmacological strategies}

With the exception of one small placebo-controlled study in which pindolol (2.5 mg TID) demonstrated an augmenting effect in fluoxetine $(20 \mathrm{mg}$ /day)-resistant panic patients (Hirschmann et al 2000), no placebo-controlled studies have yet been done to validate the effectiveness of switching compounds within or between classes or of augmenting a drug with a compound of another class.

However, in treatment resistant patients, different strategies can be suggested (Mathew et al 2001; Bandelow et al 2002):

1. an augmentation with a compound belonging to another class of drug (TCA, BDZ, pindolol);

2. a switch to MAOI;

3. a use of drugs, investigated in preliminary studies, such as venlafaxine, mirtazapine, nefazodone, reboxetine or valproate.

A combination of an SSRI with imipramine or clomipramine (recommended by the ICGDA, WFSBP, NICE and CPA guidelines) requires careful monitoring because some SSRIs increase TCA plasma levels through inhibition of cytochrome P450.

An augmentation with a BDZ (recommended by ICGDA, WFSBP and CPA guidelines) may offer some advantage in patients resistant to an adequate trial with an SSRI or TCA, particularly in those with severe anxiety and insomnia. However, this combination is problematic in long-term treatment, mainly because of the difficulty of discontinuing BDZ, and in patients with substance addiction who may abuse BDZ.

A switch to a MAOI (recommended by ICGDA, WFSBP and CPA guidelines) should be carefully monitored, because of their serious side-effects, the harmful consequences in association with other drugs that increase monoamines and the need for dietary restriction (Riederer et al 2004).

A switch from an antidepressant to valproate, or sometimes an augmentation with valproate, may represent an effective treatment strategy in PD patients refractory to first-line medication because of a bipolar disorder or "mood instability", whereas the effectiveness of other moodstabilizers (lithium, carbamazepine, gabapentin, lamotrigine, topiramate) in refractory PD is inconsistent or unproven (Mathew et al 2001; Bandelow et al 2002).

Recently, a few small open label trials (Hollifield et al 2005; Sepede et al 2006; Simon et al 2006), suggest that a second generation antipsychotic (olanzapine and risperidone) may be successfully used in patients with refractory PD. However, the limitations of the studies (small number of patients studied, open label design) did not allow for the drawing of conclusions regarding the effectiveness of a second generation antipsychotic in the treatment of refractory $\mathrm{PD}$, and the available guidelines do not recommend the use of this type of medication in the treatment of PD.

\section{Psychological strategies}

In the last decade, CBT is recommended (APA, NICE, BPA and CPA guidelines) in patients who show partial or no response to medications. Recent studies demonstrated that adding CBT improved the outcome in patients who failed to respond to an adequate trial of pharmacotherapy (Heldt et al 2003) and maintained a panic free status over a one year follow-up period in two thirds of patients resistant to medications (Heldt et al 2006).

Moreover, psychological therapy is probably beneficial for symptom stabilization in PD patients with personality disorders or with extensive psychological conflicts (APA 1998; Mathew et al 2001).

In order to manage patients who are refractory to medications, CBT seems to be the strategy preferred by psychiatrists: in a survey (Scott et al 1999), the addition of CBT in non-responders to a trial of an SSRI was the most popular choice (34\% of psychiatrists interviewed), preferred to add a BDZ (27\%) and to increase the dose of SSRI (26\%).

\section{How long should the treatment last?}

Even though the optimal length of pharmacotherapy is unclear, there is evidence that antipanic medication response continues while the patient is taking medication and that relapse is common after discontinuation of drugs (Ballenger 1999). To maintain full remission and to decrease the risk of relapse after stopping medication, a longer period of maintenance treatment (12-24 months) has been recommended by the ICGDA (Ballenger et al 1998).

Nonetheless, a recent study (Mavissakalian and Perel 2002) found a similar relapse rate $(37 \%)$ in patients 
who discontinued imipramine 6 or $12-30$ months after achieving a $50 \%$ improvement of pre-treatment severity or a presence of mild symptoms. This result suggests that the maintenance of benefit following treatment discontinuation may be much more influenced by the level of symptom severity before treatment discontinuation (the patients were again symptomatic when treatment was discontinued) than the duration of treatment. Such a finding underscores the importance of maximizing improvement: achieving a stable status of remission is needed before considering treatment discontinuation in order to increase the likelihood of maintaining improvement after stopping medication. Therefore, the discontinuation of medication should be considered in patients who maintain a full remission for a long period (6 months recommended by NICE and BPA guidelines and at least 12 months recommended by APA, ICGDA, WCA and CPA guidelines) and are not currently experiencing a stressful life event.

Otherwise, ICGDA (Ballenger et al 1998) recommended that treatment should be continued in patients with substantial residual symptoms, comorbid conditions, a history of severe relapse or high levels of stress due to important lifestyle changes. Patients should be reassured regarding the fact that $\mathrm{PD}$ is a condition that often requires long-term treatment.

\section{How to discontinue medication}

Patients should be informed not to stop medication abruptly, without consulting their physicians, to avoid a withdrawal syndrome. Severe withdrawal syndrome appears particularly after the abrupt discontinuation of BDZ (Schweizer and Rickels 1998), but symptoms of discontinuation can also follow a tapering period of a SSRI or TCA compound that is too short (Warner et al 2006). Therefore, a slow tapering of medications (4-6 months) should be recommended by ICGDA (Ballenger et al 1998).

\section{Pharmacological treatment of PD in special populations Children and adolescents}

Anxiety disorders are not easy to assess in children and adolescents and many methodological problems can reduce the reliability of the data (Silverman and Ollendick 2005), particularly the results of studies investigating PD in children.

However, epidemiological studies in community samples suggest that panic attacks are frequently observed in children and adolescents: panic attacks were observed in 3\% of youths aged from 9 to 17 years (Goodwin and Gotlib 2004) and PD was diagnosed in $10 \%-12 \%$ of clinically referred children and adolescents (Masi et al 2000; Doerfler et al 2006). Nevertheless, recommendations for the treatment of PD in youths are supported by much less evidence than in adults. Until now, only anecdotal reports or small studies (less than 20 subjects) have been published about treatment of PD in children and adolescents (Murphy et al 2000; Masi et al 2006), and showed that a SSRI compound could be safely and effectively used.

Therefore, the use of SSRI in youths needs to be more consistently demonstrated by large studies using a more powerful methodology (double-blind, controlled treatment). However, as observed in the treatment of obsessivecompulsive disorder, the use of SSRI compounds seems promising in the treatment of PD in children and adolescents. Nevertheless, a risk associated with use of SSRI drugs in children and adolescents must be evaluated. A meta-analysis, carried out by the US FDA on 24 placebo-controlled studies on antidepressants (mainly used in the treatment of major depression and obsessive-compulsive disorder), found an increased risk of suicide attempts (relative risk of 1.95; CI 95\% 1.28-2.98) in subjects treated with antidepressants (particularly paroxetine and venlafaxine) compared with subjects receiving placebo (data available on website http:// www.fda.gov/ohrms/dockets/ac/04/slides/2004-4065S1_ 08_FDA-Hammad_files/frame.htm). Therefore, the use of antidepressants must be carefully evaluated in children and adolescents with PD.

Although anecdotal reports have also been published about the safe and effective use of BDZ in the treatment of children and adolescents with PD (Masi et al 2006), BDZ are not recommended in the treatment of these subjects to avoid somnolence, impairment of cognitive function and dependence.

\section{Pregnant women}

The prevalence of PD ranged from $1.2 \%$ to $2.0 \%$ in pregnant women (Ross and McLean 2006). The onset of PD occurs in a minority of women during pregnancy, whereas in most women the pregnancy can affect the course of PD. The available data on this topic are conflicting, due to their large dispersion: in fact, the rate of women or pregnancies with an unchanged pregravid severity of symptoms ranged from $18 \%$ to $80 \%$, an increased severity from $4 \%$ to $33 \%$ and an improvement of symptoms in $10 \%-74 \%$ of cases (Ross and McLean 2006). However, the hypothesis that the pregnancy is a "well-being period" for women affected by PD is not supported by these data, even though a recent study 
(Bandelow et al 2006) found that the panic manifestations of PD were significantly lower during pregnancy than during a non-pregnancy period.

In the last decade, even though no medication recommended for the treatment of PD is licensed for the administration in pregnant women, and PD can be treated without medications using CBT, the use of a SSRI compound in pregnancy is widespread in clinical practice, as demonstrated by data collected by the Finnish and Swedish Medical Birth Registers (Malm et al 2005; Källén and Olausson 2006). In fact, nearly all the available SSRI compounds (at the moment data are not available on escitalopram) are largely used in pregnant women (1782 women in the Finnish Register and 5123 women in the Swedish register), even within the first trimester of pregnancy (1398 women in the Finnish Register and all the 5123 women in the Swedish register), at a time when the drugs may exert their possibly teratogenic effect. The available data show that the frequency of major malformations (a malformation that severely impaired function or that needed a surgical correction of the affected organ) was similar in women treated with SSRI compounds and in women without such a treatment $(4.2 \%$ vs $3.5 \%$ in the Finnish Register) and that the risk of any malformation did not increase in women treated with SSRI (OR 0.87; CI 95\% 0.76-1.01 in Swedish Register).

Nevertheless, in Swedish Register (Källén and Olaussoen 2006) an excess risk of cardiac defects (mainly atrial or ventricular septum defects) (OR 2.22; CI 95\% 1.39-3.55) was observed in births of women exposed to paroxetine (but not to other SSRI compounds) during the first trimester of pregnancy. This finding confirms the previous reports (Wogelius et al 2005; the GlaxoSmithKline advisory, available on web site www.gsk.ca/en/health_info/ PAXIL_PregnancyDHCPL_E-V4.pdf), which induced the US FDA and the Health Canada to recommend adding this information in the Warnings section of prescribing information of paroxetine. Recently, the American College of Obstetricians and Gynecologists (2006) recommends to avoid the use of paroxetine in pregnant women or in women planning to become pregnant.

However, a recent study (Berard et al 2006) suggests that only the use of paroxetine at a dose higher than $25 \mathrm{mg} /$ day in the first trimester is associated with an increased risk of major malformations $(\mathrm{OR}=2.23 ; 95 \% \mathrm{CI}=1.19-4.17)$ or an increase in cardiac defects $(\mathrm{OR}=3.07 ; 95 \% \mathrm{CI}=$ 1.00-9.42).

In late pregnancy, the administration of SSRI exposes the newborns to the following two risks:
1. about $30 \%$ of them may present a withdrawal syndrome which, however, rarely is severe enough to require treatment (Moses-Kolko et 2005; Sanz et al 2005);

2. an increased risk of persistent pulmonary hypertension in newborns of mothers taking fluoxetine (OR 6.1; CI 95\% 2.2-16.8) (Chambers et al 2006). However, this serious condition was uncommon, since about $99 \%$ of women exposed to this class of compound in late pregnancy did not deliver newborns affected by this condition.

Concerning the cognitive and behavioral effect of the in-utero exposure to antidepressants, the available data suggest that fluoxetine, taken during pregnancy, does not adversely affect cognition, language development or temperament of preschool and early school children. (Nulman et al 2002)

During pregnancy, the use of BDZ is not recommended (American Academy of Pediatrics 2000, Iqbal et al 2002; Eberhard-Gran et al 2005), because the administration of $\mathrm{BDZ}$ in the first trimester increases the risk of cleft lip/palate, and in late pregnancy induces a withdrawal syndrome or a "floppy baby syndrome" (muscular hypotonia, low Apgar scores, hyperthermia, impaired response to cold) in BDZdependent newborns.

In conclusion, the risk-benefit assessment of the use of medication in pregnant women, particularly during the first and third trimester of pregnancy, must be discussed with the woman and her family and an informed consent should be obtained.

\section{Breastfeeding women}

During post-partum, the frequency of PD increases compared to pregnancy (1.4\%-3.9\% of women), and a worsening of pregravid symptom severity more likely occurs, as observed in $20 \%-63 \%$ of women or post-partum periods (Bandelow et al 2006; Ross and McLean 2006).

In the post-partum period, the use of a SSRI must be carefully evaluated, because the newborns are exposed to medications through breast milk. Even though the available data concerning the effects of exposure of breastfeeding infants to a SSRI drug are reassuring, and breastfeeding should not be generally discouraged in women using SSRI (Weissman et al 2004; Berle et al 2004; Eberhard-Gran et al 2006), in clinical practice most women prefer to discontinue breastfeeding when a pharmacotherapy is needed to avoid a potential danger for the infant. However, when the mother chooses to take medication while breastfeeding, fluoxetine and citalopram should not be the drugs of first choice, because the long plasma half-life of the former and 
the detectable plasma level in the newborns of the latter may cause adverse effects in the infants (Eberhard-Gran et al 2006). Obviously, a non-pharmacological treatment can be recommended in women who chose breastfeeding and prefer to avoid medications.

\section{Conclusions}

The available data on the evidence-based efficacy of the pharmacological treatment in PD suggest the following indications to clinicians:

1. a SSRI compound is the first choice medication, for the beneficial side-effect profile of this class of drugs.

2. the medication should be started at a low dosage to prevent side effects and then increased until the therapeutic dose is reached;

3. in the first weeks of treatment, a BDZ can be usefully associated to a SSRI compound to rapidly improve symptoms and to mitigate the "activation" side effects of the SSRI medication;

4. the medication should be continued to achieve, when possible, a complete remission of symptoms and thereafter maintained for at least 12 months;

5. the medication should be discontinued slowly to prevent the onset of a withdrawal syndrome.

In spite of the fact that the aforementioned suggestions are recommended by all available international guidelines, some clinical problems in the treatment of PD are difficult to manage because of the lack of reliable data. Specifically, the management of partial-response or non-response is not well established because only a few studies addressed this topic with a rigorous methodology. Also, the treatment of PD in children or adolescents and in pregnant or breastfeeding women was only evaluated in observational studies. Therefore, clinicians should consider all precautions when they are asked to treat PD in these patients.

Moreover, strategies are needed to provide effective care to people with PD in the general population, since only a minority of them receives any mental health treatment.

\section{References}

American Academy of Pediatrics. 2000. Use of psychoactive medication during pregnancy and possible effects on the fetus and newborn. Pediatrics, 105:880-7.

American College of Obstetrician and Gynecologists. 2006. Treatment with selective serotonin reuptake inhibitors during pregnancy. Obstet Gynecol, 108:1601-3.

Ampollini P, Marchesi C, Signifredi R, et al. 1999. Temperament and personality features in patients with major depression, panic disorder and mixed conditions. J Affect Disord, 52:203-7.
[APA] American Psychiatric Association. 1998. Practice guidelines for the treatment of patients with panic disorder. Am J Psychiatry, 155(Suppl 5):1-34.

[APA] American Psychiatric Association. 2000. Diagnostic and stastical manual of mental disorders. Fourth edition, text revised. Washington, DC: American Psychiatric Association

Bakker A, van Balkom AJ, Spinhoven P, et al. 2002. SSRIs vs. TCAs in the treatment of panic disorder: a meta-anlsysis. Acta Psychiatr Scand, 106:163-7.

Baldwin DS, Anderson IM, Nutt DJ, et al. 2005. Evidence-based guidelines for the pharmacological treatment of anxiety disorders: recommendations from the British Association of Psychopharmacology. J Psychopharmacol, 19:567-96.

Ballenger JC. 1997. Panic disorder in the medical setting. J Clin Psychiatry, 58(Suppl 2):13-17.

Ballenger JC. 1999. Clinical guidelines for establishing remission in patients with depression and anxiety. J Clin Psychiatry, 60(Suppl 22):29-34.

Ballenger JC. 2001. Treatment of anxiety disorders to remission. $J$ Clin Psychiatry, 62(Suppl 12):5-9.

Ballenger JC, Davidson JRT, Lecrubier Y, et al. 1998. Consensus statement on panic disorder from the international consensus group on depression and anxiety. J Clin Psychiatry, 59(Suppl 8):47-54.

Bandelow B, Sojka F, Broocks A, et al. 2006. Panic disorder during pregnancy and postpartum period. Eur Psychiatry, 21:495-500.

Bandelow B, Zohar J, Hollahder E, et al. 2002. World Federation of Societies of Biological Psychiatry (WFSBP). Guidelines for the pharmacological treatment of anxiety, obsessive-compulsive and posttraumatic stress disorders. World J Biol Psychiatry, 3:171-99.

Barbey JT, Roose SP. 1998. SSRI safety in overdose. J Clin Psychiatry, 59(Suppl 15):42-8.

Barlow DH, Gorman JM, Shear MK, et al. 2000. Cognitive-behavioural therapy, imipramine, or their combination for panic disorder: a randomized controlled trial. JAMA, 283:2529-36.

Berard A, Ramos E, Rey E, et al. 2006. First trimester exposure to paroxetina and risk of cardiac malformations in infants: the importance of dosage. Birth Defects Res B Dev Reprod Toxicol, 80:18-27.

Berle JO, Steen VM, Aamo TO, et al. 2004. Breastfeeding during maternal antidepressant treatment with serotonin reuptake inhibitors: infant exposure, clinical symptoms and cytichrome p450 genotypes. $J$ Clin Psychiatry, 65:1228-34.

Bradwejn J, Ahokas A, Stein DJ, et al. 2005. Venlafaxine extended-release capsules in panic disorder: flexible-dose, double-blind, placebocontrolled study. Br J Psychiatry, 187:352-9.

Bruce SJ, Vasile RG, Goisman RM, et al. 2003. Are benzodiazepines still the medication of choice for patients with panic disorder with or without agoraphobia? Am J Psychiatry, 160:1432-8.

[CPA] Canadian Psychiatric Association. 2006. Clinical practice guidelines. Management of anxiety disorders. Can J Psychiatry, 51 (8 suppl 2): 9S-91S.

Chambers CD, Hernandez-Diaz S, Van Mater LJ, et al. 2006. Selective serotonin-reuptake inhibitors and risk of persistent pulmonary hypertension of the newborn. $N$ Engl J Med, 354:579-87.

Cowley DS, Ha EH, Roy-Byrne PP, et al. 1997. Determinants of pharmacologic treatment failure in panic disorder. J Clin Psychiatry, 58:555-61.

Davidson JRT. 1998. The long-term treatment of panic disorder. $J$ Clin Psychiatry, 59(Suppl 8):17-21.

den Boer JA. 1998. Pharmacotherapy of panic disorder: differential efficacy from a clinical viewpoint. $J$ Clin Psychiatry, 59(Suppl 8):30-6.

Doerfler LA, Connor DF, Volungis AM, et al. 2006. Panic disorder in clinically referred children and adolescents. Child Psychiatry Hum Dev, in press.

Doyle A, Pollack MH. 2003. Establishment of remission criteria for anxiety disorders. J Clin Psychiatry, (Suppl 15):40-5.

Doyle A, Pollack MH. 2004. Long-term management of panic disorder. J Clin Psychiatry, 65(Suppl 5):24-8. 
Dreessen L, Arntz A. 1998. The impact of personality disorders on treatment outcome of anxiety disorders: best-evidence synthesis. Behav Res Ther, 36:483-504.

Eaton WW, Anthony JC, Romanoski A, et al. 1998. Onset and recovery from panic panic disorders in the Baltimore Epidemiologic Catchment Area follow-up. Br J Psychiatry, 173:501-7.

Eberhard-Gran M, Esklid A, Opjordsmoen S. 2005. Treating mood disorders during pregnancy: safety considerations. Drug Saf, 28:695-706.

Eberhard-Gran M, Esklid A, Opjordsmoen S. 2006. Use of psychotropic medications in treating mood disorders during lactation: practical recommendations. CNS Drugs, 20:187-98.

Goddard AW, Brouette T, Almai A, et al. 2001. Early coadministration of clonazepam with sertraline for panic disorder. Arch Gen Psychiatry, 58:681-6.

Goisman RM, Warshaw MG, Peterson LG, et al. 1994. Panic, agoraphobia, and panic disorder with agoraphobia. Data from a multicenter anxiety disorders study. J Nerv Ment Dis, 182:72-9.

Goodwin RD, Gotlib IH. 2004. Panic attacks and psychopathology among youth. Acta Psychiatr Scand, 109:216-21.

Goodwin RD, Roy-Byrne P. 2006. Panic and suicidal ideation and suicide attempts: results from the National Comorbidity Survey. Depress Anxiety, 23:124-32.

Grant BF, Hasin DS, Stinson FS, et al. 2005. Co-occurrence of 12-month mood and anxiety disorders and personality disorders in the US: results from the national epidemiologic survey on alcohol and related conditions. J Psychiatr Res, 39:1-9.

Greenblatt DJ, von Moltke LL, Harmatz JS, et al. 1998. Drug interactions with newer antidepressant: role of human cytochromes P450. J Clin Psychiatry, 59(Suppl 15):19-27.

Heldt E, Manfro GG, Kipper L, et al. 2003. Treating medication-resistant panic disorder: predictors of cognitive-behaviour therapy in a Brazilian public hospital. Psychother Psychosom, 72:43-8.

Heldt E, Manfro GG, Kipper L, et al. 2006. One-year follow-up of pharmacotherapy-resistant patients with panic disorder treated with cognitivebehaviour therapy: outcome and predictors of remission. Behav Res Ther, 44:657-65.

Hischmann S, Dannon PM, Iancu I, et al. 2000. Pindolol augmentation in patients with treatment-resistant panic disorder: A double-blind, placebo controlled trial. J Clin Psychopharmacol, 20:556-9.

Hollifield M, Thompson PM, Ruiz JE, et al. 2005. Potential effectiveness and safety of olanzapine in refractory panic disorder. Depress Anxiety, 21:33-40.

Iqbal MM, Sobhan T, Ryals T, 2002. Effects of commonly used benzodiazepines on the fetus, the neonate, and nursing infant. Psychiatr Serv, 53:39-49.

Kallen B, Olausson PO. 2006. Antidepressant drugs during pregnancy and infant congenital heart defect. Reprod Toxicol, 21:221-2.

Katerndahl DA, Realini JP. 1995. Where do panic sufferers seek care? J Fam Pract, 40:237-43.

Katschnig H, Amering M. 1998. The long-term course of panic disorder and its predictors. J Clin Psychopharmacol, 18(Suppl 2):6-11.

Kessler RC, Berglund P, Demler O, et al. 2005. Lifetime prevalence and ageof-onset distributions of DSM-IV disorders in the National Comorbidity Survey Replication. Arch Gen Psychiatry, 62: 593-602.

Kessler RC, Chiu WT, Demler O, et al. 2005. Prevalence, severity, and comorbidity of 12-month DSM-IV disorders in the National Comorbidity Survey Replication. Arch Gen Psychiatry, 62:617-27.

Kessler RC, Stang P, Wittchen H, et al. 1998. Lifetime panic-depression comorbidity in the National Comorbidity Survey. Arch Gen Psychiatry, 55:801-08.

Khan A, Kolts RL, Rapaport MH, et al. 2005. Magnitude of placebo response and drug-placebo differences across psychiatric disorders. Psychol Med, 35:743-9.

Klein DF. 1964. Delineation of two drug-responsive anxiety syndromes. Psychopharmacologia, 17:397-408.

Kroenke K. 2003. Patients presenting with somatic complaints: epidemiology, psychiatric comorbidity and management. Int J Methods Psychiatr Res, 12:34-43.
Lecrubier Y. 1998. The impact of comorbidity on the treatment of panic disorder. J Clin Psychiatry, 59(Suppl 8):11-14.

Malm H, Klaukka T, Neuvonen PJ. 2005. Risks associated with selective serotonin reuptake inhibitors in pregnancy. Obstet Gynecol, 106:1289-96.

Marchesi C, Brusamonti E, Giannini A, et al. 2001. The use of an emergency ward by patients with depressive or anxiety disorders: a one year follow-up study. Int J Psychiatr Med, 31:265-75.

Marchesi C, Brusamonti E, Borghi C, et al. 2004. Anxiety and depressive disorders in an emergency department ward of a general hospital: a control study. Emerg Med J, 21:175-9.

Marchesi C, Cantoni A, Fontò S, et al. 2005. The effect of pharmacotherapy on personality disorders in panic disorder: a one year naturalistic study. $J$ Affect Disord, 89:189-94.

Marchesi C, Cantoni A, Fontò S, et al. 2006a. Predictors of symptom resolution in Panic Disorder after one year of pharmacological treatment: a naturalistic study. Pharmacopsychiatry, 39:60-5.

Marchesi C, Cantoni A, Fontò S, et al. 2006b. The effect of temperament and character on response to Selective Serotonin Reuptake Inhibitors in Panic Disorder. Acta Psychiatr Scand, 114: 203-10.

Marchesi C, De Panfilis C, Cantoni A, et al. 2006. Personality Disorders and response to medication treatment in Panic Disorder: a one year naturalistic study. Prog Neuro-Psychopharmacol Biol Psychiatry, 30:1240-45

Maron E, Shlik J. 2006. Serotoni function in panic disorder: important, but why? Neuropsychopharmacology, 31:1-11.

Masi G, Favilla L, Mucci M, et al. 2000. Panic disorder in clinically referred children and adolescents. Child Psychiatry Hum Dev, 31:139-51.

Masi G, Pari C, Millepiedi S. 2006. Pharmacological treatment options for panic disorder in children and adolescents. Expert Opin Pharmacother, 7:545-54.

Massion AO, Dyck IR, Shea MT, et al. 2002. Personality disorders and time to remission in generalized anxiety disorder, social phobia, and panic disorder. Arch Gen Psychiatry, 59:434-40.

Mathew SJ, Caplan JD, Gorman JM. 2001. Management of treatmentrefractory panic disorder. Psychopharmacol Bul, 35:97-110.

Mavissakalian MR, Perel JM. 2002. Duration of imipramine therapy and relapse in panic disorder with agoraphobia. J Clin Psychopharmacol, 22:294-9.

Mitte K. 2005. A meta-analysis of the efficacy of psycho- and pharmacotherapy in panic disorder with and without agoraphobia. $J$ Affect Disord, 88:27-45.

Moses-Kolko EL, Bogen D, Perel J, et al. 2005. Neonatal signs after late in utero exposure to serotonin reuptake inhibitors: literature review and implications for clinical applications. JAMA, 293:2372-83.

Murphy TK, Bengston MA, Y Tan J, et al. 2000. Selective serotonin reuptake inhibitors in the treatment of paediatric anxiety disorders: a review. Int Clin Psychopharmacology, 15(Suppl 2):s47-s63.

[NICE] National Institute for Clinical Excellence. 2004. Anxiety: management of anxiety (panic disorder, with or without agoraphobia, and generalised anxiety disorder) in adults in primary, secondary and community care. Clinical Guideline 22: December 2004. National Collaborating Centre for Primary Care. Available on web site: www.nice.org.uk.

Neumeister A, Daher RJ, Charney DS. 2005. Anxiety disorders: noradrenergic neurotrasmission. Handb Exp Pharmacol, 169:205-23.

Ninan PT, Dunlop BW. 2005. Neurobiology and etiology of panic disorder. $J$ Clin Psychiatry, 66(Suppl 14):3-7.

Nulman I, Rovet J, Stewart DE, et al. 2002. Child development following exposure to tricyclic antidepressants or fluoxetine throughout fetal life: a prospective, controlled study. Am J Psychiatry, 159:1889-95.

Ongur D, Farabaugh A, Iosifescu DV, et al. 2005. Tridimensional personality questionnaire factors in major depressive disorder: relationship to anxiety disorder comorbidity and age of onset. Psychother Psychosom, 74:173-8.

Otto MW, Tuby KS, Gould RA, et al. 2001. An effect-size analysis of the relative efficacy and tolerability of serotonin selective reuptake inhibitors for panic disorder. Am J Psychiatry, 158:1989-92. 
Ozkan M., Altindag A, Toni A, et al. 2005. Comorbid personality disorders in subject with panic disorder: Do personality disorders increase clinical severity? Compr Psychiatry, 46:20-6.

Pollack MH. 2005. The pharmacotherapy of panic disorder. J Clin Psychiatry, 66(Suppl 4):23-7.

Pollack MH, Allgulander MD, Bandelow B, et al. 2003. WCA Recommendations for the Long-Term Treatment of Panic Disorder. CNS Spectr, 8(suppl 1): 17-30.

Pollack MH, Simon NM, Worthington JJ, et al. 2003. Combined paroxetine and clonazepam treatment strategies compared to paroxetine monotherapy for panic disorder. J Psychopharmacol, 17:276-82.

[RANZCP] Royal Australian and New Zealand College of Psychiatrists Clinical Practice Guidelines Team for Panic Disorder and Agoraphobia. 2003. Australian and New Zealand clinical practice guidelines for the treatment of panic disorder and agoraphobia. Aust N Z J Psychiatry, 37:641-56.

Riederer P, Lachenmayer L, Laux G. 2004. Clinical application of MAOinhibitors. Curr Med Chem, 11:2033-43.

Roose SP, Miyazaki M. 2005. Pharmacologic treatment of depression in patients with heart disease. Psychosom Med, 67(Suppl 1):s54-7.

Rosenbaum JF, Pollack MH, Pollock RA. 1996. Clinical issues in the long-term treatment of panic disorder. J Clin Psychiatry, 57(Suppl 10):44-8.

Ross LE, McLean LM. 2006. Anxiety disorders during pregnancy and the postpartum period: a systematic review. J Clin Psychiatry, 67:1285-98

Sanz EJ, De-las-Cuevas C, Kiuru A, et al. 2005. Selective serotonin reuptake inhibitors in pregnant women and neonatal withdrawal syndrome: a database analysis. Lancet, 365:482-7.

Sareen J, Cox BJ, Afifi TO, et al. 2005. Anxiety disorders and risk for suicidal ideation and suicide attempts: a population- based study of adults. Arch Gen Psychiatry, 62:1249-57.

Schweizer E, Rickels K. 1998. Benzodiazepine dependence and withdrawal: a review of the syndrome and its clinical management. Acta Psychiat Scand, 98(Suppl 393):95-101.

Scott EL, Pollack MH, Otto MW, et al. 1999. Clinician Response to treatment Refractory Panic Disorder: A Survey of Psychiatrists. J Nerv Dis, 187:755-7.

Sepede G, De Berardis D, Gambi F, et al. 2006. Olanzapine augmentation in treatment-resistant panic disorder: a 12-week, fixed dose, open-label trial. J Clin Psychopharmacol, 26:45-9.

Silverman WK, Ollendick TH. 2005. Evidence-Based Assessment of Anxiety and Its Disorders in Children and Adolescents. $J$ Clin Child Adolesc Psychol, 34:380-411.
Simon NM, Fischmann D. 2005. The implications of medical and psychiatric comorbidity with panic disorder. J Clin Psychiatry, 4:8-15.

Simon NM, Hoge EA, Fischmann D, et al. 2006. An open-label trial of risperidone augmentation for refractory anxiety disorders. $J$ Clin Psychiatry, 67:381-5.

Slaap BR, den Boer JA. 2001. The prediction of nonresponse to pharmacotherapy in panic disoder: a review. Depress Anxiety, $14: 112-22$.

Susman J, Klee B. 2005. The role of high-potency benzodiazepines in the treatment of panic disorder. Prim Care Companion J Clin Psychiatry, $7: 5-11$.

Taylor C, King R, Margraf J, et al. 1989. Use of medication and in vivo exposure in volunteers for panic disorder research. Am J Psychiatry, 146:1423-6.

Vickers K, Mc Nally RJ. 2004. Panic disorder and suicide attempt in the National Comorbidity Survey. Depress Anxiety, 113:582-91.

Warner CH, Bobo W, Warner C, et al. 2006. Antidepressant discontinuation syndrome. Am Fam Physician, 74:449-56.

Weissman MM, Bland RC, Canino GJ, et al. 1997. The cross-national epidemiology of panic disorder. Arch Gen Psychiatry, 54:305-9.

Weissman AM, Levy BT, Hartz AJ, et al. 2004. Pooled analysis of antidepressant level in lactating mothers, breast milk, and nursing infants. Am J Psychiatry, 161:1066-78.

Wogelius P, Norgaard M, Muff Munk E, et al. 2005. Maternal use of selective serotonin reup-take inhibitors and risk of adverse pregnancy outcomes. Pharmacoepidemiol Drug Saf, 14:S72.

Yonkers KA, Ellison JM, Shera DM, et al. 1996. Description of antipanic therapy in a prospective longitudinal study. J Clin Psychopharmacol, 16:223-32.

Zajeca J, Tracy KA, Mitchell S. 1997. Discontinuation symptoms after treatment with serotonin reuptake inhibitors: a literature review. J Clin Psychiatry, 58:291-7.

Zamorski MA, Albucher RC. 2002. What to do when SSRIs fail: eight strategies for optimizing treatment of panic disorder. Am Fam Physician, 66:1477-84.

Zimmermann P, Wittchen HU, Hofler M, et al. 2003. Primary anxiety disorders and the development of subsequent alcohol use disorder: a 4-year community study of adolescents and young adults. Psychol Med, 33:1211-22.

Zwanzger P, Rupprecht R. 2005. Selective GABAergic tretament for panic? Investigations in experimental panic induction and panic disorder. J Psychiatry Neurosci, 30:167-75. 